Research Article

\title{
Diagnosis and Classification Decision Analysis of Overheating Defects of Substation Equipment Based on Infrared Detection Technology
}

\author{
Zhigang Shi, Yunlong Zhao $\mathbb{D}^{\mathrm{D}}$, Zhanshuang Liu, Yanan Zhang, and Le Ma \\ State Grid Qinghai Maintenance Company, Xining 810021, China \\ Correspondence should be addressed to Yunlong Zhao; ylzhao.sgqh@yahoo.com
}

Received 9 September 2021; Revised 1 November 2021; Accepted 16 November 2021; Published 17 December 2021

Academic Editor: Bai Yuan Ding

Copyright (c) 2021 Zhigang Shi et al. This is an open access article distributed under the Creative Commons Attribution License, which permits unrestricted use, distribution, and reproduction in any medium, provided the original work is properly cited.

\begin{abstract}
Substation equipment is not only the main part of the power grid but also the essential part to ensure the development of the national economy and People's Daily life of one of the important infrastructure. How to ensure its normal operation and find the sudden failure has become a hot issue to be solved urgently. For thermal fault diagnosis needs to classify and identify different power equipment first, this paper designed an SVM infrared image classifier, which can effectively identify three types of common power equipment. The classifier extracts HOG features from the infrared images of power equipment processed by the above segmentation and combines them with SVM multiclassification to achieve the purpose of improving the recognition accuracy. The experiment uses the classifier to identify three kinds of equipment, and the results show that the comprehensive recognition accuracy of the classifier is more than $95.3 \%$, which is better than the traditional classification method and meets the demand for classification accuracy. In this paper, the traditional method of relative temperature difference is improved by using the temperature data of the infrared image, which can automatically judge the thermal failure level of electric power equipment. Experiments show that the diagnosis system designed in this paper can classify faults and give treatment suggestions while judging whether there are thermal faults for three types of power equipment, which verifies the feasibility and effectiveness of the substation infrared diagnosis technology designed in this paper.
\end{abstract}

\section{Introduction}

With the rapid development of the social economy, the requirements for the safety and reliability of substation equipment are constantly improving. Whether the electrical equipment can operate safely and reliably has become the key to the normal operation of the power system. Substation equipment in a long-term working state will inevitably have faults, and the thermal fault is the most common fault of electrical equipment, which is manifested in abnormal temperature inside the equipment: such as poor contact heating caused by accumulated pollution on the surface of electrical equipment, insufficient clamping force of isolating switch finger that leads to overheating of the switch knifeedge, and so on. Therefore, detecting and diagnosing the temperature change of substation equipment plays an important role in the normal operation of the power grid [1].
Infrared thermal image detection technology has the advantages of no contact, high-temperature measurement efficiency, wide detection range, and so on, which can effectively improve the reliability of results and effectively improve the safety factor. In current substations, infrared thermal image detection technology has been widely used for fault detection of power equipment. Infrared thermal imaging technology can judge whether there is a thermal fault in the current running equipment in time when the power equipment is running, In order to quickly locate potential dangerous areas, it is convenient to take control measures before equipment failure and operation interruption, reduce the possibility of users' power interruption caused by substation power outage detection and troubleshooting, prevent premature failure, delay the service life of power equipment, and reduce expensive power outage and downtime [2]. The infrared thermal imaging technology currently applied in the 
field of substation equipment diagnosis; whether there are thermal defects in power equipment can be judged by manual analysis after a professional holds a thermal infrared imager to collect equipment images. This method will consume a lot of time when it is urgent to troubleshoot a large number of equipment and will also lead to diagnostic errors due to inexperience or fatigue diagnosis of staff. In view of the shortcomings of the above-mentioned manual analysis, it is necessary to use an intelligent identification and diagnosis method to judge whether there is a thermal fault in substation equipment so as to realize a rapid diagnosis and investigate the power accidents caused by potential thermal faults. Through the processing of infrared image denoising and segmentation of substation equipment, the thermal fault judgment method and infrared power equipment image recognition method are introduced, which lays a foundation for automatic recognition and thermal fault diagnosis of power equipment based on infrared image [3].

Overheating of electrical equipment is a common phenomenon. If the overheating area can be found accurately and directly, the fault range will be directly reduced. Therefore, we propose a new overheated area detection algorithm. This algorithm uses Ostu algorithm to segment the general area of equipment, then refines the target area by edge, and uses the FCM clustering algorithm to accurately divide the overheated part. After verification, the algorithm has practical value [4]. The demand for electricity has always been huge, and the country is constantly expanding the scale of the power grid to meet the transmission capacity. Obviously, the expansion of the scale also increases the load of power equipment; the frequency of faults also increases; and the workload of troubleshooting is increasing day by day. We combine patrol robot, support vector machine (SVM), and MATLAB software for simulation analysis and compare them with information entropy method. Finally, we apply the two methods to a project for periodic observation at the same time and find that the patrol robot with support vector machine has high efficiency [5]. Pressure monitoring technology can be used to monitor the status of oil-free power equipment because the insulation overheating of oilfree power equipment will lead to pressure change. Taking the change trend of pressure bearing index as an example, the pressure rises from $1 \mathrm{kPa}$ to $3 \mathrm{kPa}$ within 50 hours, and the pressure rises obviously, which shows that the pressure monitoring technology can monitor local overheated insulation defects online, and this technology is a hot spot for this problem at present [6].

Parallel conductors in AC systems often have the problem of current imbalance, which will lead to overheating and aging, and promote the occurrence of faults. Then, the fault diagnosis system is necessary. Based on a large section aluminum conductor, a spacer, and two terminal substation connectors, we have a Hall effect sensor system, which has the real-time and contactless characteristics. Through experiments, it can accurately detect the fault and current at the same time, which shows its effect and feasibility [7]. The heat dissipation problem of transformer in substation building must be solved under the condition of unsatisfactory ventilation. We use CFD to simulate the heat value around the equipment, and the model should consider the expansion fin to enhance the heat dissipation. We put forward three schemes, all of which consider the air outlet position. Finally, it is explained that choosing the appropriate air inlet and outlet position in the substation plays a key role in avoiding overheating of the transformer [8].

\section{Infrared Image Segmentation of Substation Equipment}

2.1. Intuitionistic Fuzzy Clustering Algorithm. Intuitionistic fuzzy set theory is widely used to deal with fuzzy and uncertain data and ambiguous relationships and has been applied in many fields. There is a normalized relationship among three members in an intuitionistic fuzzy set. As long as the values of any two members in the three are known, the values of the remaining members can be obtained by using the normalized relationship. Common intuitionistic fuzzy sets are usually represented by a membership function and intuitionistic index, and nonmembership can be generated by specific functions. The clustering algorithm is also used in the power system in infrared image segmentation, in which $\mathrm{K}$-means clustering is a commonly used feature with low complexity and fast convergence [9-12]. However, the noise processing effect is not ideal, and it is widely used in infrared images.

The segmentation method based on fuzzy clustering divides image data into several classes by a certain criterion, which makes the similarity between the same classes maximum and the similarity between different classes minimum. In the actual clustering process, the segmentation method based on fuzzy clustering uses a membership function to classify image data, which represents the degree of the pixel belonging to a certain region in image segmentation. Fuzzy C-means clustering algorithm is an unsupervised algorithm. Its core idea is to update the clustering center and membership function continuously until the best clustering center cut-off is obtained. The core idea of this algorithm in image segmentation is to get the best image classification result through the best clustering center. In the process of image segmentation with fuzzy C-means clustering algorithm, human interference is not needed, and the inherent fuzziness of the image can be effectively processed. An intuitionistic fuzzy clustering method based on local information is proposed [9].

2.2. Fuzzy C-Means Clustering. Fuzzy C-means clustering (FCM) is a process of dividing the elements of a set into several self-similar classes, which divides a set of elements into several classes, and the elements in each class are similar $[13,14]$. Elements in the same class have great similarities, while elements in different classes have great differences. FCM proposed by Dunn in 1973 is generalized by Bezdek J.C. Its objective function is defined as follows:

$$
J_{m}=(U, X)=\sum_{j=1}^{c} \sum_{k=1}^{n} u_{j k}^{m} d^{2}\left(x_{k}, v_{j}\right),
$$


where $U$ represents fuzzy membership matrix, $c$ represents the number of clustering centers, $n$ represents the number of pixels in the image, $x_{k}$ denotes the $k$-th data point, $v_{j}$ denotes the $j$-th cluster, $u_{j k}$ represents the fuzzy membership degree of the $k$-th pixel sample to the $j$-th cluster center, and $d^{2}\left(x_{k}, v_{j}\right)$ is expressed as the distortion degree by Euclidean distance as follows:

The following restrictions are met:

$$
\sum_{j=1}^{c} u_{j k}=1
$$

where $d^{2}\left(x_{k}, v_{j}\right)$ is represented as the distortion by $\mathrm{Eu}$ clidean distance as follows:

$$
d^{2}\left(x_{k}, v_{j}\right)=x_{k}-v_{j}^{2}
$$

When the function reaches the extreme value, the algorithm has the best effect, so the minimum values of $J_{m}(U, X)$ calculated by Lagrange number multiplication are as follows:

$$
\begin{aligned}
u_{j k} & =\left[\sum_{i=1}^{c} \frac{x_{k}-v_{j}}{x_{k}-v_{i}}\right]^{-2 / m-1}, \\
v_{j} & =\frac{\sum_{k}^{n} u_{j k}^{m} x_{k}}{\sum_{k}^{n} u_{j k}^{m}}
\end{aligned}
$$

2.3. Intuitionistic Fuzzy C-Means Clustering (IFCM). Main ideas of FCM algorithm [15, 16] described in the characteristic function (whose value can only take 0 or 1 ) to any membership function in the range of $[0,1]$. However, the FCM algorithm cannot describe the membership degree between right and wrong. In order to solve this problem, an intuitionistic FCM algorithm is proposed.

In intuitionistic fuzzy set theory, "hesitation degree" is put forward, that is, it represents the status of neutral attributes, and depicts the fuzzy information in objective reality more completely and accurately. The $n$-dimensional data set $X$ is represented by the following formula:

$$
A=\left\{\left(x, u_{A}(X), v_{A}(X)\right), x \in X\right\},
$$

where $u_{A}(X)$ is the membership degree, $v_{A}(X)$ is a nonmembership degree, and the corresponding function is shown in the following formula:

$$
u_{A}(X): x \longrightarrow[0,1] ; v_{A}(X): x \longrightarrow[0,1] .
$$

If $x \in X, x$ does not belong to $u_{A}(X)$ and $v_{A}(X)$. Then, when the condition $0 \leq u_{A}(X),+(X) \leq 1$ is satisfied, the hesitation degree is described by the following formula:

$$
\pi_{A}(x)=1-u_{A}(X)-v_{A}(X) .
$$

I represents the image, $\mathrm{X}$ represents the image pixels, and the gray value is $[0,1]$. The image is normalized as shown in the following formula:

$$
u_{A}(X)=\frac{x-I_{\min }}{I_{\max }-I_{\min }}
$$

where $I_{\max }$ and $I_{\min }$ represent the maximum gray value and the minimum gray value in the pixel, respectively. Sugeno calculates the nonmembership degree of intuitionistic fuzzy sets by using negative function as follows:

$$
v_{A}(X)=\frac{1-u_{A}(x)}{1+\lambda u_{A}(x)}
$$

where $\lambda$ is a normal number; this ensures that the sum of membership degree and nonmembership degree will not be greater than 1 .

Furthermore, let $d: \operatorname{IFS}(X) \times \operatorname{IFS}(X) \longrightarrow[0,1]$. If $d$ is a distance measure, the following attributes should be satisfied:

(1) $0 \leq d(A, B) \leq 1$

(2) If $A=B$ then $d(A, B)=0$

(3) $d(A, B)=d(B, A)$

(4) If $A \subseteq B \subseteq C$ and $A, B, C \in \operatorname{IFSs}(X)$, $d(A, B) \leq d(A, C)$ and $d(B, C) \leq d(A, C)$

then

The normalized distances of $A$ and $B$ are shown in the following formula:

$$
d_{I F S}(A, B)=\left\{\frac{1}{2 n} \sum_{i=1}^{n}\left[\left(u_{A}\left(x_{i}\right)-u_{B}\left(x_{i}\right)\right)^{2}+\left(v_{A}\left(x_{i}\right)-v_{B}\left(x_{i}\right)\right)^{2}+\left(\pi_{A}\left(x_{i}\right)-\pi_{B}\left(x_{i}\right)\right)^{2}\right]\right\}^{1 / 2} .
$$

Formula (10) is improved to obtain the following formula:

$$
\widetilde{d}\left(A_{1}, A_{2}\right)=\left\{\frac{1}{2 n} \sum_{i=1}^{n}\left[p_{12}\left(u_{A 1}\left(x_{i}\right)-u_{A 2}\left(x_{i}\right)\right)^{2}+q_{12}\left(v_{A 1}\left(x_{i}\right)-v_{A 2}\left(x_{i}\right)\right)^{2}+\rho\left(A_{1}, A_{2}\right)\left(\pi_{A 1}\left(x_{i}\right)-\pi_{A 2}\left(x_{i}\right)\right)^{2}\right]\right\}^{1 / 2} .
$$


The weight of $p_{12}, q_{12}$, and $\rho\left(A_{1}, A_{2}\right)$ can be adjusted according to the application data. The IFCM function definition is shown in the following formula:

$$
J_{\mathrm{IFCM}}=\sum_{J=1}^{C} \sum_{k=1}^{n} u_{j k}^{m} d_{\mathrm{IFS}}^{2}\left(x_{k}, v_{j}\right),
$$

where $d_{\mathrm{IFS}}^{2}$ is the intuitionistic ambiguity distance. The iterative formula of membership degree and clustering centroid based on the Lagrange multiplier method is as follows:

$$
\begin{aligned}
u_{j k} & =\left[\sum_{i=1}^{c} \frac{d_{\mathrm{IFS}}\left(x_{k}, v_{j}\right)}{d_{\mathrm{IFS}}\left(x_{k}, v_{i}\right)}\right]^{-2 / m-1}, \\
u_{A}\left(v_{j}\right) & =\frac{\sum_{k=1}^{n} u_{j k}^{m} u_{A}\left(x_{k}\right)}{\sum_{k=1}^{n} u_{j k}^{m}}, \\
v_{A}\left(v_{j}\right) & =\frac{\sum_{k=1}^{n} u_{j k}^{m} v_{A}\left(x_{k}\right)}{\sum_{k=1}^{n} u_{j k}^{m}}, \\
\pi_{A}\left(v_{j}\right) & =\frac{\sum_{k=1}^{n} u_{j k}^{m} \pi_{A}\left(x_{k}\right)}{\sum_{k=1}^{n} u_{j k}^{m}} .
\end{aligned}
$$

In the IFCM algorithm, the cluster center $v_{j}$ is expressed as $v_{j}=u_{A}\left(v_{j}\right) v_{A}\left(v_{j}\right) \pi_{A}\left(v_{j}\right)$. The convergence of the IFCM algorithm is proved by formulas (13)-(16), and the local minimum of the objective function is proved by formula (13).

\section{Automatic Classification of Power Equipment Based on the Infrared Image}

3.1. Feature Extraction of the Infrared Image. Feature is a collection of the essence and characteristics of a certain object relative to other objects, so feature extraction can be regarded as using a mapping relationship to describe highdimensional image features [17] from low-dimensional spatial features. Generally speaking, images have their own characteristics that can be distinguished from other images, such as color, edge information, texture features, and so on. Common image features are as follows:

\section{(1) Color characteristics}

Color feature, as a global feature with high efficiency and good effect, expresses the surface characteristics of the target object. Half of it is represented as a three-dimensional space, and the other is represented by three color parameters, such as RGB, XYZ, and other color spaces. Color features are based on pixels and insensitive to the change of image direction and size, so they cannot describe the local changes of target objects in the image.

(2) Texture features

Texture feature, as a common statistical feature in image recognition, is generated by color spatial transformation. Although there is no completely unified theoretical concept at present, it is similar to color feature, and it is also an external feature to express the target object in the image. Texture feature extraction, which is widely used in practice, generally includes statistical method and model method. In the actual texture extraction, the resolution will change, and there will be a large error at this time.

(3) Spatial relationship characteristics

Spatial relation feature, as a feature index in image space, means that there is a certain relationship between the position or direction of the segmented object in image space. Spatial relation features are generally used when it is necessary to enhance the ability of the object of the expressed image. However, it is easily affected by the position translation, rotation change, and size change of the image or a certain area of the image in actual operation, which will make the spatial relationship feature unable to accurately express the scene information of the image. Therefore, it will be combined with other feature extraction methods in practice to improve the accuracy of the expression of the image scene.

(4) Shape characteristics

Shape features are generally divided into contour and region features. The contour feature describes the outer boundary of the object in the image, and the region feature covers all the object regions in the image. Shape feature extraction is not affected by image position transformation or scale transformation. Common shape feature extraction, such as moment invariants, wavelet description, and so on.

For the infrared image studied in this paper, color information maps the temperature data of the target object, instead of the color characteristics of the object itself. Therefore, its contrast is not high. The texture of the target object cannot be clearly displayed. It is also important to note that in the actual operation of collecting infrared images of power equipment, the image will inevitably change in spatial position, so using the above three features, that is, color, texture, and spatial relationship features, to describe the target equipment in infrared images is not in line with the actual feature extraction requirements. Compared with other features, shape features can better identify the power equipment in infrared images. Therefore, the Hu moment of the invariant moment is used to recognize the shape features of power equipment in the infrared image.

3.2. Infrared Image Recognition. Image recognition is one of the practical applications of pattern recognition in digital image processing. Generally, image segmentation and feature extraction are carried out, and after image analysis, a certain feature in the selected image is recognized and then classified. The specific flow is shown in Figure 1. In this paper, image acquisition refers to the use of infrared thermal imager for power equipment image acquisition and import into the computer for the next processing operation. Image segmentation is to segment the target image, that is, the faulty power equipment from the image background. 


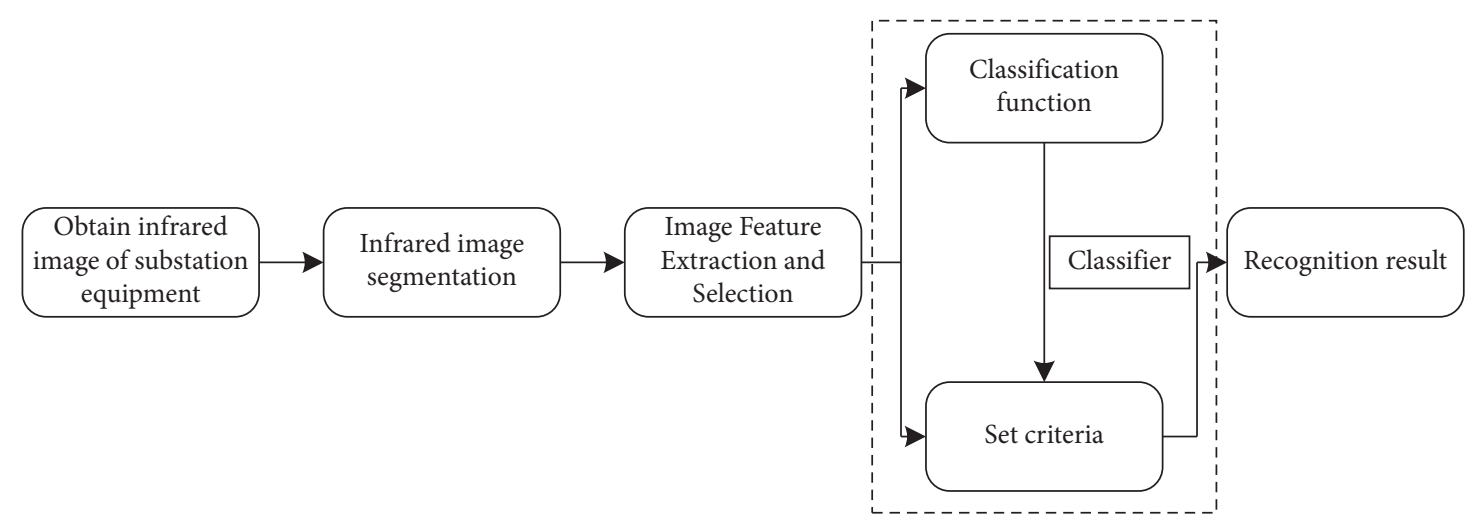

Figure 1: Flow chart of image recognition.

Feature extraction and selection are to process the image information and then extract the features that can describe the essence of the target object segmented from the image. The classifier uses the features extracted in the previous step to classify and recognize the image features through appropriate functions and evaluation criteria.

Up to now, many algorithms for image recognition have been widely used, such as artificial neural network recognition, fuzzy recognition, and template matching. In this paper, a support vector machine algorithm based on statistical theory is used to classify and recognize infrared images of substation equipment. The classification function mainly classifies images according to the features extracted from images. Generally, minimum distance classification is adopted, and there are machine learning methods, such as SVM, KNN, SVM, BPNN, CNN, and transfer learning. According to the set standard, the image features are classified so as to realize the classification function.

\subsection{Feature Extraction Based on Directional Gradient} Histogram. The feature based on directional gradient histogram is a feature descriptor for object recognition, which uses directional gradient histogram of the local area of the statistical image to form features. In the field of image classification, using HOG features combined with SVM classification has been widely used [18].

HOG feature extraction is a good way to describe the image or shape of the local region of the image through the distribution of gradient or edge direction density. The process is shown in Figure 2.

The specific process is described by Figure 2:

(1) Firstly, the infrared image of power equipment is normalized by gamma ray, and the image is divided into smaller units, which are called cell units. In order to unify the overall standard, images are generally converted into gray images, and the gray values are set in the range of $[0,1]$.

(2) The gradient value is calculated. The gradient operator $[-1,0,1]$ is used to convolve the image, and the gradient component in the horizontal $x$-axis direction is obtained. Similarly, the gradient components perpendicular to the $y$-axis direction are obtained by using this operator, and the gradient size and direction of each pixel in the digital image are calculated by using these two gradient components.

(3) Secondly, the directional gradient histogram is constructed. Each cell is divided into nine uniform directional blocks; then each pixel is mapped to a fixed angle range; and the gradient directional histogram of the cell is obtained, which contains the corresponding nine-dimensional feature vector of the cell.

(4) In block normalized gradient histgram, the cells are combined into an interval, and the HOG features of all cells in this interval are obtained by connecting the eigenvectors in series. The overlapping of different intervals means that the different results of each cell feature will be expressed in the final feature vector, so the feature vector in the block should be normalized.

(5) Finally, the HOG features are collected. All overlapping blocks are collected by HOG features, and the final combined feature vectors are used for classification.

3.4. Substation Equipment Classification Based on SVM. In practical applications, two types of classifiers widely used are artificial neural networks and support vector machines. Both of them use the training set to obtain the recognition system model to classify the data set to be recognized. The difference is that the former is based on statistical theory and simulates the complex regular network built by the human brain neural network. The BP neural network model [19] shown in Figure 3 is most commonly used in practical applications.

In the latter, the input space is changed into a highdimensional space by a specific function so as to transform the complex nonlinear relationship into a linearized relationship. When using a neural network to summarize complex laws, the recognition accuracy of data to be recognized is generally improved by adding a large number of known result data sets. However, although the recognition error rate decreases, the corresponding network scale and complexity will gradually increase, that is to say, a small 


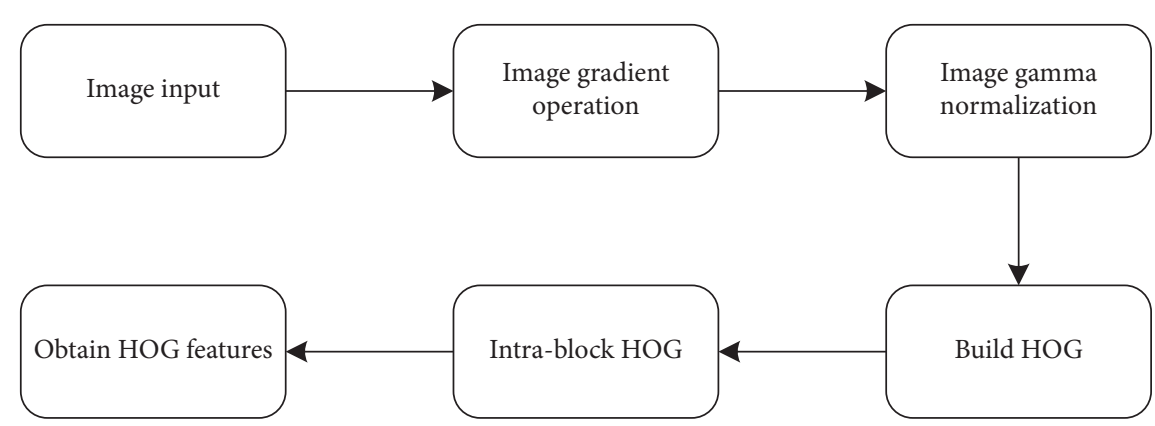

FIgURe 2: Flowchart of HOG feature extraction.

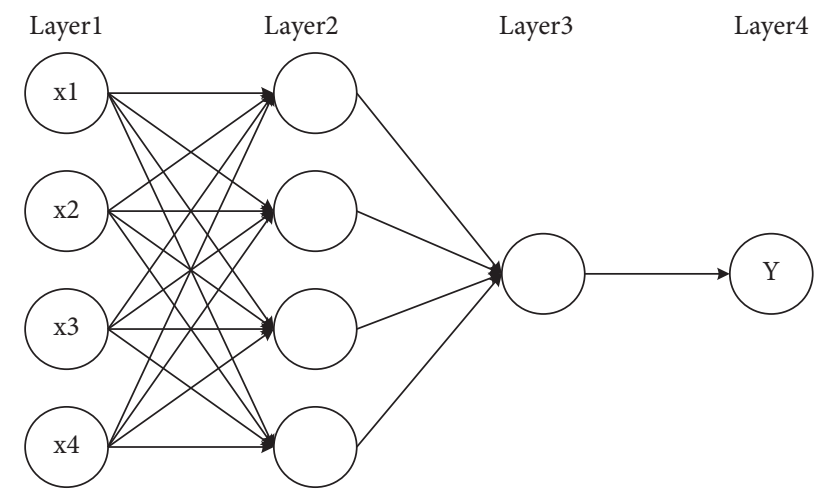

FIGURE 3: Basic model of the neural network.

number of training sets cannot ensure a high recognition rate. However, in practice, it is difficult to collect an infinite number of data samples, which makes the error rate infinitely reduced, and it is difficult to achieve ideal classification results. And blindly compressing the redundant areas of training data will lead to overfitting, which is also one of the representative shortcomings of neural networks in practice.

Comparatively speaking, a support vector machine has better classification ability when it cannot obtain a considerable number of data sets. Support vector machine is good at solving the classification problem of small samples and nonlinear data, and the global optimal solution obtained by optimization method can prevent the problem of overlearning. Therefore, this paper chooses the SVM algorithm for the automatic classification of infrared images of power equipment.

SVM is different from ANN in that it can learn from small samples and has the advantage of high learning efficiency. SVM is widely used in regression and classification research directions and has a high accuracy for nontraining samples.

As shown in Figure 4, it is a schematic diagram of SVM classification $[20,21]$ under the condition that two-dimensional data are linearly separable. It can be seen from the diagram that if two types of samples are completely divided by a linear function, they are linearly separable samples; otherwise, they are nonlinear separable samples.

Real and empty points represent two kinds of samples in a two-dimensional plane, in which the positive example label is $l$ and the negative example label is -1 , and the samples are linearly separable. $L$ is the final linear classifier; and $L 1$ and

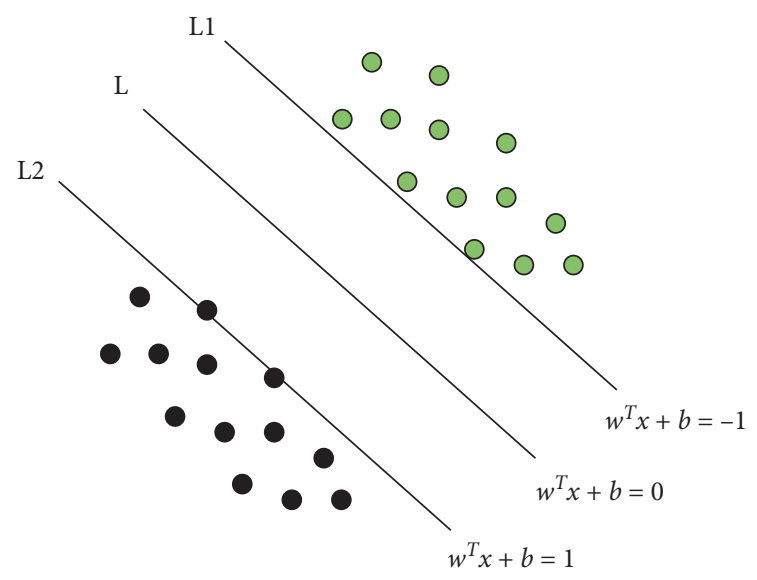

FIGURE 4: SVM linear classification model.

$L 2$ are the straight lines where the support vectors are located, parallel to $L$; and their intervals are classification intervals. To get the optimal classification line is to get a classification line that can divide the data into two categories with the largest interval.

The above-mentioned SVM theory is only suitable for binary classification, but the actual situation is often like the power equipment classification studied in this paper. In order to extend SVM to solve multiclass classification problems, the "one-to-many" method of indirect method, "one-to-one" method, and multiclass support vector machine of direct method are generally used.

\section{Thermal Fault Diagnosis of Power Equipment Based on the Infrared Image}

The operation of substation equipment is easily affected by the external environment, such as artificial construction, temperature, humidity, and electric field interference. In the long run, some problems such as poor contact of power equipment will inevitably occur, which will lead to local heating of equipment and cause thermal failure. If it is not found in time and hidden dangers are not eliminated in advance, it is easy to cause major accidents such as power failure and even explosion. Infrared diagnosis is used to detect the infrared radiation signal of power equipment. After the temperature distribution map is obtained, it is preliminarily judged whether the equipment has fault signs through analysis. On the basis of image segmentation and 
recognition, this chapter designs an infrared diagnosis method of power equipment based on the combination of relative temperature difference method and temperature data and realizes the basic thermal fault diagnosis function of the infrared image of power equipment.

4.1. Infrared Thermal Fault Diagnosis Principle. The wavelength of the infrared spectrum is between 0.75 and $1,000 \mu \mathrm{m}$, which is outside the visible red light region, so it is also called the infrared ray. In the natural environment, because the molecules and atoms in any object move irregularly, they will continuously radiate energy outward, so infrared rays exist most widely compared with other electromagnetic radiation.

There are too many external interference factors and complicated conditions when studying actual objects, so people set up a physical model with maximum radiation power, that is, blackbody, whose incident radiation absorptivity is a fixed value 1 under all conditions. Although the blackbody hypothesized here is only an ideal model for studying infrared radiation, it is used as a standard to measure the radiation amount, and its thermal radiation law shows that the thermal radiation has a relationship with wavelength and temperature, so it is widely used in the precision calibration of infrared equipment and other fields.

There is a close relationship between the energy of an object and the motion of molecules and atoms inside it. The more violent the irregular motion inside the object, the greater the energy contained in the object and the higher its own temperature. On the contrary, for stationary particles, their temperature is absolute zero, and there is no radiant energy that will be emitted.

4.2. Infrared Thermal Fault Diagnosis Means. Infrared detection technology, which is a noncontact detection technology, will inevitably bring the defect of low-temperature measurement accuracy in fault detection of substation equipment, which will directly affect the detected temperature value. In order to improve the accuracy of infrared diagnosis of power equipment, researchers and professional inspectors put forward three applicable diagnosis methods after a large number of measurement practices:

(1) Surface temperature judgment method

After measuring the surface temperature of power equipment with a thermal imager, the overheated part of the equipment is judged according to the relevant data standard of Common Technical Requirements of High-Voltage Switchgear and Control Equipment Standard, combined with the current environmental temperature condition and equipment load degree. This judgment method is simple and effective, but because of the influence of external factors on the accuracy of temperature value, there is a situation that the fault of small temperature rise cannot be accurately judged.

(2) Relative temperature difference judgment method
The relative temperature difference judgment method measures the temperature difference between two corresponding temperature measuring points of two devices that meet the same or similar basic states such as category and temperature and calculates the temperature rise at the higher point. Finally, the relative temperature difference $\sigma_{t}$ defined by the relative temperature difference method is calculated by the ratio of the two, and its expression is shown in the following equation:

$$
\begin{aligned}
\sigma_{t} & =\frac{\tau_{1}-\tau_{2}}{\tau_{1}} \times 100 \%=\frac{\left(T_{1}-T_{0}\right)-\left(T_{2}-T_{0}\right)}{T_{1}-T_{0}} \times 100 \% \\
& =\frac{T_{1}-T_{2}}{T_{1}-T_{0}} \times 100 \%
\end{aligned}
$$

where $\sigma_{t}$ is the relative temperature difference of the measured point; $\tau_{1}$ and $T_{1}$ are the temperature rise (unit: $\mathrm{K}$ ) and temperature (unit: $\mathrm{K}$ ) of the starting point, respectively, while $\tau_{2}$ and $T_{2}$ are the temperature rise and temperature of the normal measured point respectively; and $T_{0}$ is the ambient temperature. When the ambient temperature of the equipment is low or the measured temperature is within the normal value specified in GB736-90, it cannot be directly determined that the equipment does not have an abnormal heating problem, but it can be determined in the actual infrared diagnosis that the probability of thermal failure increases with the increase of ambient temperature. By calculating the relative temperature difference of equipment, this method is generally applicable to diagnose the fault of equipment caused by current or voltage.

(3) Similar comparison method

similar comparison method, as its name implies, is a method to compare and judge the temperature difference of similar equipment with the same working state and external environment including temperature and noise, which is used for fault diagnosis of voltage-induced equipment.

4.3. Diagnosis and Result Analysis of Substation Equipment. In this paper, the relative temperature difference is calculated by the temperature data measured by the infrared thermal imager, and then whether there is a fault in suspected power equipment is diagnosed, and the severity of equipment fault is graded. This chapter uses the relative temperature difference method of power equipment combined with the fault diagnosis standard of common power equipment and carries out thermal fault diagnosis and fault classification experiments for three common substation equipment.

4.3.1. Selection of Thermal Fault Diagnosis Methods. Through the definition of the relative temperature difference method in the previous section, it can be determined that 
relative to other thermal fault diagnosis methods, such as surface temperature judgment method and similar comparison method, the relative temperature difference method can accurately judge whether there is a thermal fault in the measured power equipment. When a load of power equipment increases or a short circuit occurs, the relative temperature difference method can judge whether the power equipment is faulty or not by measuring the temperature of the heating part of the equipment and calculating the change of temperature rise, so it can judge the severity of the electrical equipment fault, so it is widely used in the thermal fault diagnosis of power equipment. The relative temperature difference is judged as shown in Table 1.

Attention should be paid to the use of the relative temperature difference judgment method in actual operation:

(1) When calculating the relative temperature difference, the three values measured, that is, the normal position, the suspected fault position, and the external environment, must be the values measured by the same instrument at the same time or at short intervals. It should be noted that the ambient temperature must be the value obtained by measuring the equipment in nonoperation by the thermal imager, and the atmospheric temperature cannot be simply measured by other methods; otherwise, the error of ambient temperature will lead to an inaccurate relative temperature difference value, which will affect the diagnosis and fault of suspected faulty equipment.

(2) Although the relative temperature difference is theoretically independent of the environment and load conditions, it is not applicable to all situations. In order to reduce the influence of solar radiation on the test results, the test value of the equipment should be carried out in the infrared environment with insufficient light as much as possible.

(3) For the fault of voltage-induced equipment, although theoretically, there is a similar relationship between the relative temperature difference and equipment loss. However, in general, the relative temperature difference method does not diagnose such faults.

4.3.2. Determination of Thermal Fault Level. In this paper, the relative temperature difference method is used to judge the fault level of the infrared image of the power equipment to be measured, and the relative temperature difference $\sigma_{t}$ of the equipment is calculated according to the expression. According to the $\sigma_{t}$ of different power equipment under different thermal fault levels in Table 1, the threshold value of the three common power equipment required in this paper is set.

Firstly, the relative temperature difference $\sigma_{1}$ of highpressure casing is determined. According to the criteria for judging the thermal defects of high-pressure casing in Table $1, \sigma_{1}$ is set: if $\sigma_{1}<0.20$, the high-pressure casing is diagnosed to be in normal operation; If $0.2 \leq \sigma_{1}<0.8$, the general fault is diagnosed; if $0.8 \leq \sigma_{1}<0.95$, serious faults will be diagnosed; and if $\sigma_{1} \geq 95$, the emergency fault is diagnosed, and the diagnosis process is shown in Figure 5.

Secondly, the isolation relative temperature difference $\sigma_{2}$ is determined, which is similar to that of a high-voltage sleeve. According to the thermal defect judgment standard of isolating switch in Table $1, \sigma_{2}$ is set: the diagnostic isolating switch is diagnosed as normal when $\sigma_{2}<0.35$ is different from $\sigma_{1}$; when $0.35<\sigma_{2}<0.8$, it is diagnosed as a general fault; when $0.8<\sigma_{2}<0.9$, it is diagnosed as a serious fault; and when $\sigma_{2} \geq 0.95$, it is diagnosed as a critical fault, and its diagnosis flow is shown in Figure 6.

In the same step as the above two types of equipment, the relative temperature difference $\sigma_{3}$ of the clamp is finally determined. $\sigma_{3}$ is set according to the judgment standard of thermal defect of the clamp in Table 1. The diagnostic criteria of relative temperature difference $\sigma_{3}$ of clamp are the same as $\sigma_{2}$, and the diagnostic flow is shown in Figure 7.

\subsubsection{Thermal Fault Diagnosis and Result Analysis. As an} important component of the substation system, high-voltage bushing plays an irreplaceable role and function, which ensures that the leads keep insulated to the ground by leading the internal leads of the transformer to the external insulating bushing of the oil tank. An infrared thermal imager can be used for daily inspection and maintenance of casing and prevent the occurrence of faults and defects.

The high-voltage bushing adjusts the lead direction and protects the lead to insulate it from the ground. When the internal overheating is serious, it will lead to the failure of the circuit connection and the breakdown accident of the dielectric, which is an important hidden danger that seriously affects the normal operation and power supply of the substation. In order to realize the thermal fault diagnosis of the high-voltage casing, according to the above processing steps, firstly, the infrared images of high-voltage casing are segmented and classified by SVM, and then the thermal fault diagnosis method combining relative temperature difference method and temperature data is used to diagnose and classify the thermal fault of the high-voltage casing.

This experiment runs under MATLAB 2020b platform, and its diagnosis results are shown in Figure 8. In the left infrared image, the stigma at the top of the casing is regarded as an abnormal hot spot. The diagnosis results show that the power equipment in the image is a high-voltage casing, and its relative temperature difference is $72.6 \%$, which belongs to general defects. It can be concluded that the classification is correct, the fault grade obtained by the relative temperature difference method is accurate, and the operation countermeasures are given.

Isolating switch, as an interrupt device in substation, mainly completes the conversion between circuit devices and is a switch breaker without a special arc-extinguishing device. Because of its lack of arc extinguishing ability, the isolating switch is generally limited to operating without load. However, if an oil circuit breaker is installed in the circuit, it can also be operated with a load under certain technical conditions. Due to a series of outage maintenance 
TABLE 1: Determination of relative temperature difference of current heating equipment.

\begin{tabular}{|c|c|c|c|}
\hline \multirow{2}{*}{ Type of equipment } & \multicolumn{3}{|c|}{ Relative temperature difference fault range } \\
\hline & General fault type & Major thermal failure & Emergency thermal failure \\
\hline SF6 circuit breaker & $\geq 20 \%$ & $\geq 80 \%$ & $\geq 95 \%$ \\
\hline Vacuum circuit breaker & $\geq 20 \%$ & $\geq 80 \%$ & $\geq 95 \%$ \\
\hline Oil-filled casing & $\geq 20 \%$ & $\geq 80 \%$ & $\geq 95 \%$ \\
\hline High-voltage switchgear & $\geq 35 \%$ & $\geq 80 \%$ & $95 \%$ \\
\hline Isolating switch & $\geq 35 \%$ & $\geq 80 \%$ & $\geq 95 \%$ \\
\hline Other conductive equipment & $\geq 35 \%$ & $\geq 80 \%$ & $\geq 95 \%$ \\
\hline
\end{tabular}

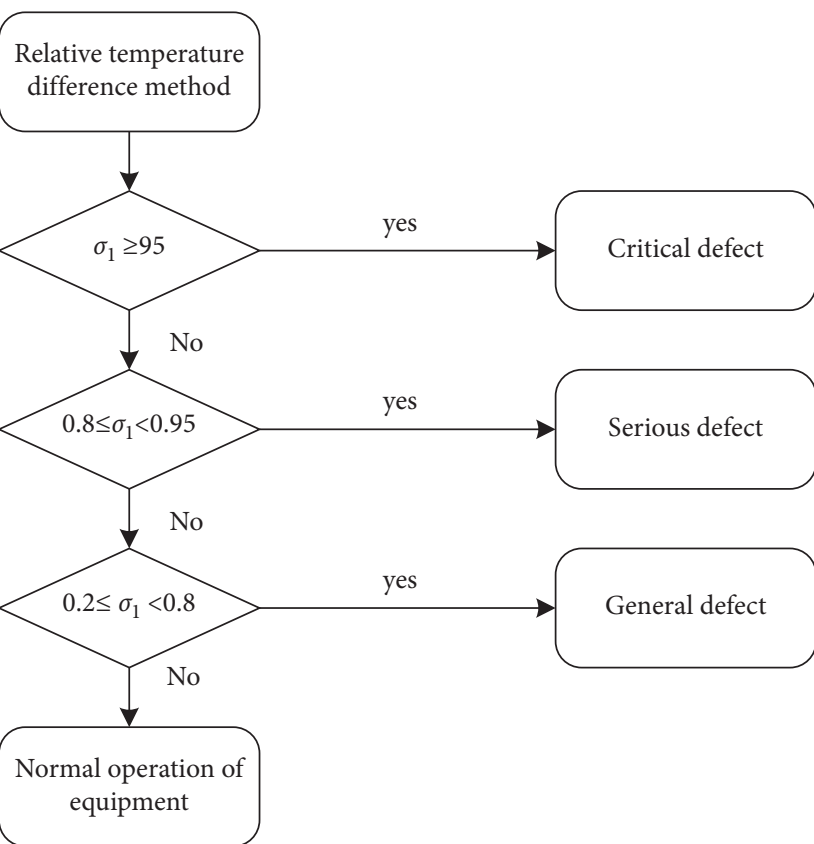

FIGURE 5: Diagnostic flow of high-pressure casing.

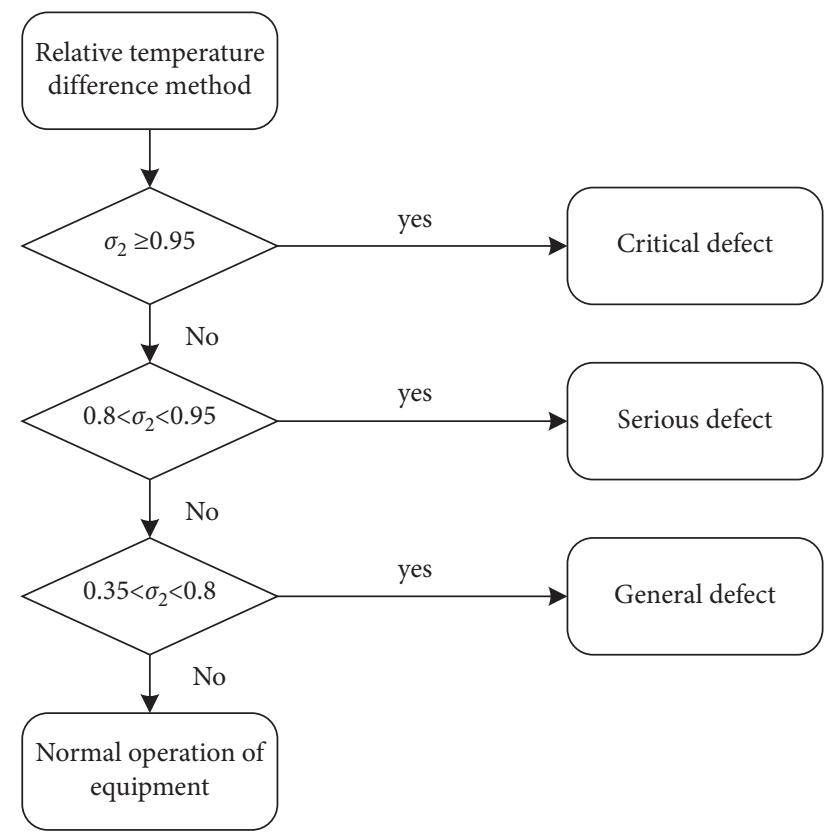

Figure 6: Diagnostic flow of disconnector.

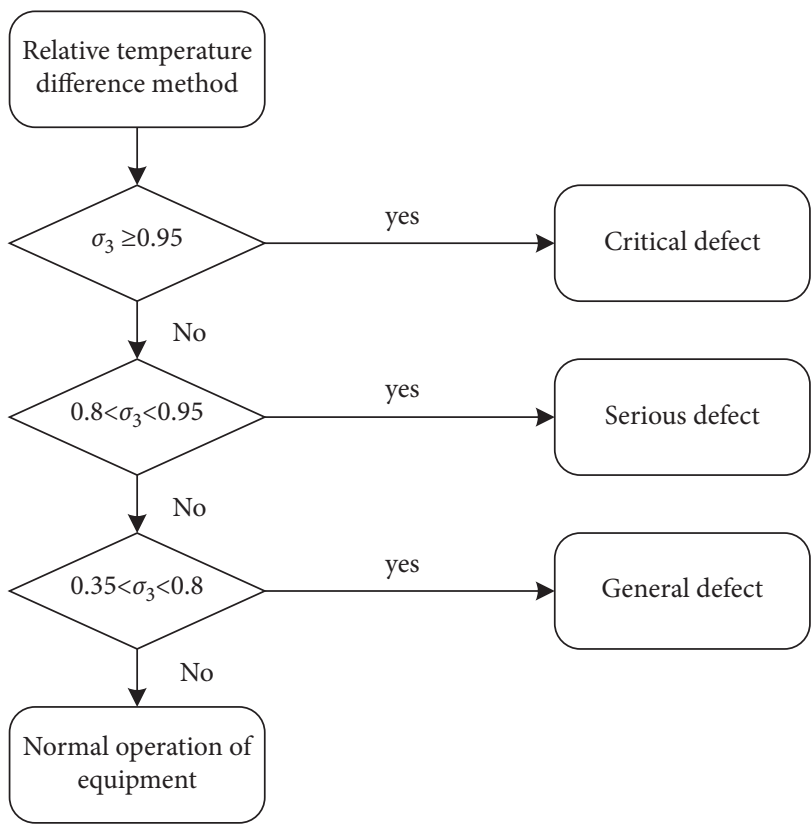

Figure 7: Diagnostic flow of device clamp.

work in the substation, the disconnector equipment operates frequently, and its conductive part is exposed to air, which leads to poor contact and overheating of equipment, so the probability of thermal failure is higher than the other two types.

This study is also carried out under MATLAB 2020b platform, and its diagnosis results are shown in Figure 9, in which the contact heads are divided into hot spots. The diagnosis results show that the power equipment in the image is an isolating switch, and its relative temperature difference is $80.2 \%$, which is a serious defect. It can be concluded that the classification results are correct, the fault grade is accurate, and the relative treatment opinions are given.

As a common connecting component in the substation transmission line, the thermal fault of wire clamp generally has the following reasons: improper operation of hydraulic wire clamp during hydraulic installation leads to a thermal fault; bolt clamp in daily operation or maintenance of bolt fastening is not in place; or vibration phenomenon in operation leads to poor contact of clamp caused by thermal failure.

The diagnosis results of the disconnector under MATLAB 2020b platform in this study are shown in Figure 10. 

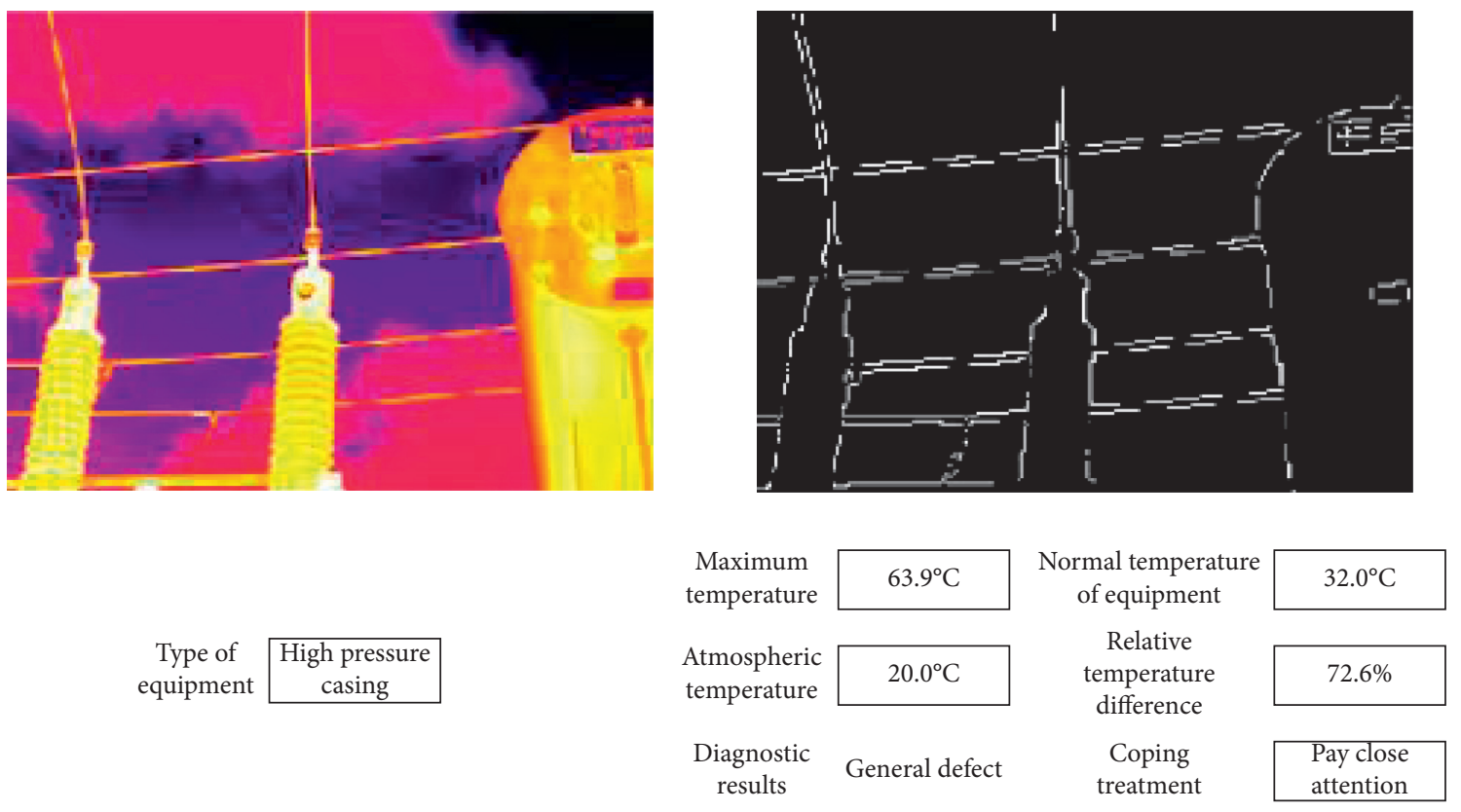

Figure 8: Thermal fault diagnosis results of high-voltage casing.
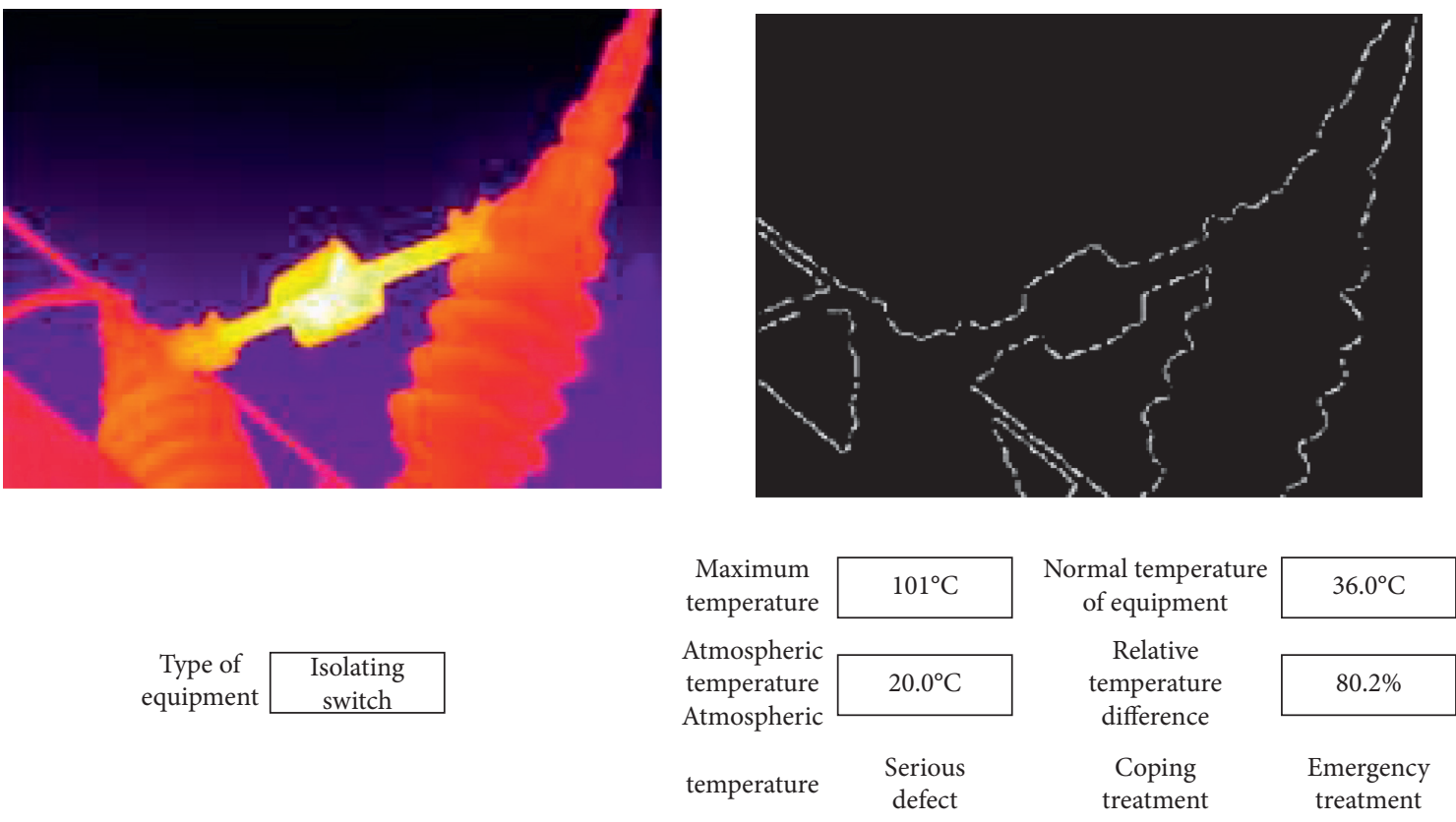

FIGURE 9: Disconnector thermal fault diagnosis result.

It can be seen from the above figure that the joint part of the wire clamp is obviously heated in the infrared image, and the diagnosis result shows that the power equipment in the image is an equipment wire clamp, and its relative temperature difference is $88.3 \%$, which is a serious defect. It can be concluded that the classification result is correct, and the accurate fault level is also given, and there are relative treatment opinions.

4.3.4. Recognition Accuracy of Classification Experiment. In this experiment, the infrared image classification recognizer based on HOG-SVM proposed in this paper is used to classify and identify substation equipment. The classification experiment flow is shown in Figure 11.

One hundred and fifty infrared images of high-voltage bushing, 100 isolating switches, and 100 wire clamps in substation equipment are selected. Among them, 100 infrared images of high-voltage bushing, 50 infrared images of isolating switches, and 50 infrared images of wire clamps are taken as training samples of HOG-SVM. The remaining 50 infrared images of high-voltage bushing, 50 isolating switches, and 50 wire clamps are used as the images to be classified by the HOG-SVM substation equipment classifier.

The classification and recognition experiment of substation equipment in this paper is implemented under the 

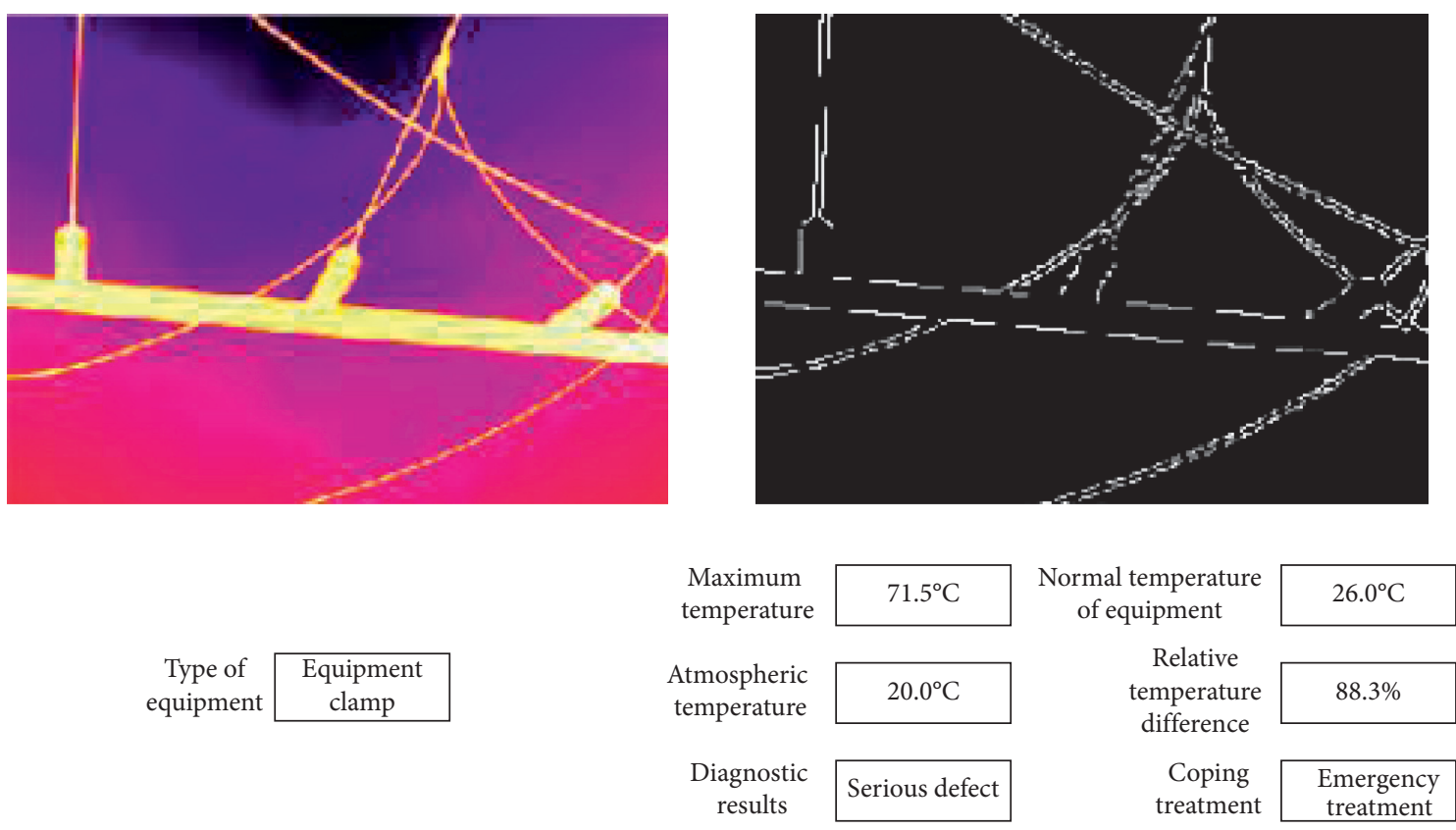

Figure 10: Thermal fault diagnosis results of equipment clamp.

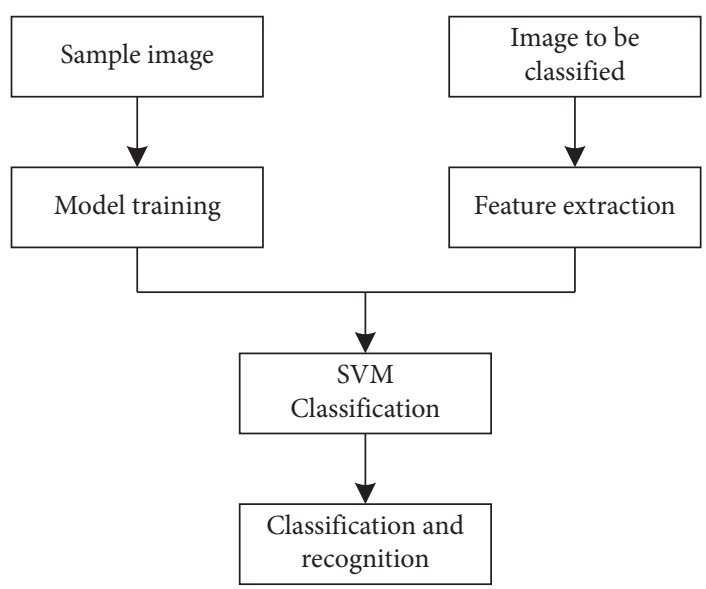

FIGURE 11: SVM infrared image classification process.

LIBSVM toolbox of MATLAB 2016b platform. The specific classification steps are as follows:

(1) Firstly, the infrared image preprocessing and image segmentation methods given in Sections 3 and 4 are used for image denoising, image enhancement, and image segmentation of all selected experimental samples.

(2) The HOG feature extraction and SVM multiclassification training are carried out on 100 infrared images of high-voltage sleeve, 50 isolating switches, and 50 wire clamps.

(3) At last, the classification method of HOG-SVM is used to classify and identify 50 images of high-voltage bushing, isolating switch, and clamp equipment in turn.
The classification results of each device are shown in Table 2.

It can be seen from Table 2 and Figure 12 that the HOGSVM classification method is used to classify 50 infrared images of high-pressure casing, and the classification accuracy rate is $96 \%$; the classification accuracy of isolating switches is $90 \%$; and the classification accuracy of clamps is $100 \%$. Through the comprehensive analysis of three common substation equipment, the total number of correct classification can be calculated to be 143 , and the comprehensive classification accuracy of the three kinds of equipment reaches $95.3 \%$. It can be seen that the infrared image classification method of substation equipment based on HOG-SVM proposed in this study has a good classification effect. 
TABle 2: Substation equipment classification results based on HOG-SVM.

\begin{tabular}{lccc}
\hline & High-pressure casing & Isolating switch & Wire clamp \\
\hline Total number of samples & 150 & 100 & 100 \\
Number of training samples & 100 & 50 & 50 \\
Number of samples to be classified & 50 & 50 & 50 \\
Correct classification number & 48 & 45 & 50 \\
\hline
\end{tabular}

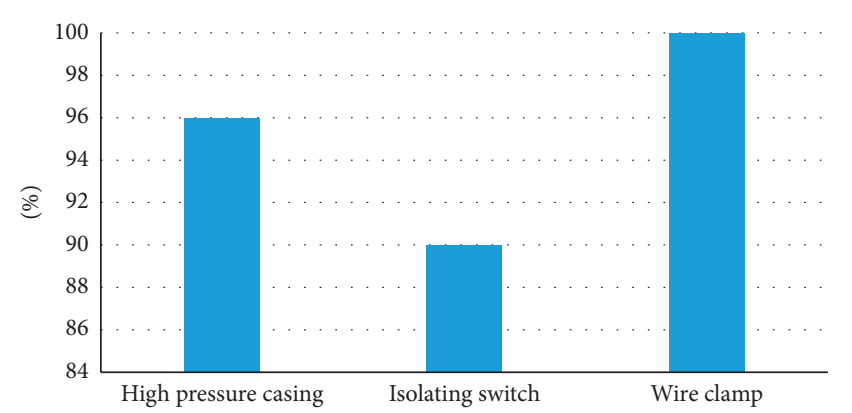

FIgURE 12: Classification accuracy.

\section{Conclusion}

After segmenting and classifying the infrared images of the equipment, this paper designs a fault diagnosis system for substation equipment based on infrared thermal image technology and realizes the accurate identification and diagnosis of thermal faults of three common power equipment, namely, high-voltage bushing, isolating switch, and equipment clamp. Based on the analysis of the features of the infrared image of power equipment in feature extraction, this paper designs a classifier for infrared power equipment image by using SVM and realizes the recognition of three kinds of power equipment, and the recognition accuracy reaches the predetermined expectation. In addition, on the basis of summarizing the types of electrical equipment faults and their causes and characteristics, in view of how to judge the thermal failure of equipment from the abnormal temperature rise of the thermal image, through the temperature difference discriminant method combined with infrared image temperature data on three types of common equipment in substations, the accurate diagnosis results and corresponding treatment suggestions are obtained, which verifies that the thermal fault diagnosis method of substation equipment designed in this paper is of practical significance.

With the rapid evolution of related infrared technology, it is believed that it will continue to exert its energy in the detection of power equipment. In the future, infrared diagnosis technology will be highly intelligent and integrated into the smart grid vigorously developed by State Grid. Infrared detection devices will be used to automatically collect infrared images, automatically process image information to diagnose faulty equipment, and upload them to the control terminal for the overall deployment of staff to carry out efficient detection and maintenance operations. In this paper, the classification and thermal fault diagnosis of substation equipment based on infrared images are basically realized. However, due to the limited time, there are still some shortcomings. For example, the number of infrared power equipment data sets collected is insufficient, which leads to the inability to classify equipment images more accurately. This is the place that needs to be improved in the future.

\section{Data Availability}

The experimental data used to support the findings of this study are available from the corresponding author upon request.

\section{Conflicts of Interest}

The authors declare that they have no conflicts of interest regarding this work.

\section{References}

[1] H. Yanjun, C. Honggang, Z. Taoyun et al., "Design of transformer secondary voltage drop on-line monitoring system based on Internet of things," IOP Conference Series: Materials Science and Engineering, vol. 677, no. 5, Article ID 052028, 2019.

[2] C. Li, Z. Yu, and M. Zhuo, "Research on fault detection method of infrared thermal imaging for power equipment based on deep learning," IOP Conference Series: Earth and Environmental Science, vol. 714, no. 4, Article ID 042045, 2021.

[3] X. Gao, H. Liu, L. Du, and T. Wu, "Study on the online status monitoring technology of intelligent substation virtual circuit based on comprehensive evaluation and recognition method," Dianli Xitong Baohu yu Kongzhi/Power System Protection and Control, vol. 47, no. 3, pp. 182-188, 2019.

[4] S. Fan, T. Li, Y. Liu, Y. Gong, and K. Yu, "Infrared imagebased detection method of electrical equipment overheating area in substation," E3S Web of Conferences, vol. 185, no. 10, Article ID 01034, 2020.

[5] X. Zhao, Z. Peng, and S. Zhao, "Substation electric power equipment detection based on patrol robots," Artificial Life and Robotics, vol. 25, no. 385-386, 2020.

[6] Q. Yu, H. Liu, Q. He, G. Zhang, Z. Zhang, and X. Zhang, "Research on pressure-based detection technology for partial overheat insulation defect of oil-less power equipment," IOP Conference Series: Earth and Environmental Science, vol. 632, Article ID 042009, 2021.

[7] A. Kadechkar, J. R. Riba, M. Moreno-Eguilaz, and J. Sanllehí, "Real-time wireless, contactless, and coreless monitoring of the current distribution in substation conductors for fault diagnosis," IEEE Sensors Journal, vol. 5, pp. 1693-1700, 2018.

[8] F. Bahri and H. Hasini, "Flow and heat dissipation analysis in transformer substation with minimal ventilation using CFD," International Journal of Engineering \& Technology, vol. 7, no. 4, pp. 327-332, 2018.

[9] P. Shang, H. Dong, X. Li, and W. Ren, "Research on fault diagnosis method of $750 \mathrm{kV}$ substation based on Bayesian network and fault recording information fusion," Journal of 
Physics: Conference Series, vol. 1550, no. 5, p. 5, Article ID 052020, 2020.

[10] S. Biricik and H. Komurcugil, "Three-level hysteresis current control strategy for three-phase four-switch shunt active filters," IET Power Electronics, vol. 9, no. 8, pp. 1732-1740, 2016.

[11] Z. Lu, T. Wang, J. Zhang, T. Jin, and S. Lu, "Research and application of substation site maintenance assistant safety management system based on computer control system," Journal of Physics: Conference Series, vol. 1574, Article ID 012112, 2020.

[12] A. Nasiri, A. Taheri-Garavand, M. Omid, and G. M. Carlomagno, "Intelligent fault diagnosis of cooling radiator based on deep learning analysis of infrared thermal images," Applied Thermal Engineering, vol. 163, Article ID 114410, 2019.

[13] F. Hu, H. Chen, and X. Wang, "An intuitionistic kernel-based fuzzy C-means clustering algorithm with local information for power equipment image segmentation," IEEE Access, vol. 8, pp. 4500-4514, 2020.

[14] M. A. Basaran, B. Simonetti, and A. A. Basaran, "Quantification of qualitative assessments using computing with words: in framework of fuzzy set theory," Soft Computing, vol. 24, no. 18, pp. 13565-13577, 2020.

[15] J. Fang, X. Song, N. Yao, and M. Shi, “Application of FCM Algorithm combined with articial neural network in TBM operation data," Computer Modeling in Engineering and Sciences, vol. 126, no. 1, pp. 397-417, 2021.

[16] M. Chen, C. Tang, M. Xu, and Z. Lei, "Binarization of optical fringe patterns with intensity inhomogeneities based on modified FCM algorithm," Optics and Lasers in Engineering, vol. 123, pp. 14-19, 2019.

[17] X. Zhao, H. Wei, H. Wang, T. Zhu, and K. Zhang, "3D-CNNbased feature extraction of ground-based cloud images for direct normal irradiance prediction," Solar Energy, vol. 181, pp. 510-518, 2019.

[18] L. Wang, J. Gui, Z. M. Lu, and C. Liu, "Fast pedestrian detection and tracking based ON vibe combined hog-svm scheme," International Journal of Innovative Computing Information and Control, vol. 15, no. 6, pp. 2305-2320, 2019.

[19] X. Zhang, T. Xue, and H. Eugene Stanley, "Comparison of econometric models and artificial neural networks algorithms for the prediction of baltic dry index," IEEE Access, vol. 7, pp. 1647-1657, 2019.

[20] G. Chen and S. Li, "Research on location fusion of spatial geological disaster based on fuzzy SVM," Computer Communications, vol. 153, pp. 538-544, 2020.

[21] G. Chen, X. Xie, and S. Li, "Research on complex classification algorithm of breast cancer chip based on SVM-RFE gene feature screening," Complexity, vol. 2020, Article ID 1342874, 12 pages, 2020 . 